\title{
Phase Quantized Metasurface Reflectors for X-band Laguerre Gaussian Vortex Beam Generation
}

\author{
Rajanikanta SWAIN, Rabindra Kishore MISHRA \\ Dept. of Electronics Science, Berhampur University, 760007 Berhampur, India \\ rks.rs.es@buodisha.edu.in, rk.elsc@ieee.org \\ Submitted July 29, 2018, / Accepted September 14, 2018
}

\begin{abstract}
From last two decades, there is an exponential growth in consumption of available bandwidth in the radio frequency spectrum. To challenge this issue, improvement in channel capacity is getting huge research attention. Laguerre-Gaussian vortex beams are one of the solutions to challenge so-called Multi-Input-Multi-Output (MIMO) technology. However, designing compact portable antennas to generate vortex beams at radio frequencies is still a challenge. We proposed two metasurface reflector models (track and sector-wise distribution) based on 3-bit phase quantized meta-element analysis to generate Orbital Angular Momentum (OAM) vortex modes. A microstrip antenna is used as a feeding element instead of the conventional horn to reduce overall reflector size. Simulated E-field distribution clarifies the spatial vortex mode behavior at X-band. Experimental results of fabricated prototypes at $9.5 \mathrm{GHz}, 10 \mathrm{GHz}$, and $10.5 \mathrm{GHz}$ agree with simulated far-fields which indicate a broadband characteristic.
\end{abstract}

\section{Keywords}

Antenna, metasurface, Orbital Angular Momentum (OAM), reflectarray

\section{Introduction}

To overcome the issue in channel capacity, advanced modulation formats like Wavelength Division Multiplexing (WDM)/Phase Division Multiplexing (PDM) are playing a significant role in communication engineering. However, the spectrum consumption which is linearly increasing with time may not be fulfilled by these techniques. Recently, the introduction of Space Division Multiplexing (SDM) and Mode Division Multiplexing (MDM) in the optical regime are independent of bandwidth, and they may hold the promise to address the issue in radio frequency. The MDM, in particular, can improve the channel capacity based on individual data-stream transmission through spatial modes. The OAM vortex beams [1] are such modal basis in which terabit communication capacity is being proposed recently
[2]. According to Maxwell's theory, energy and momentum (linear and angular) are two components of EM radiation. Moreover, angular momentum comprises spin and orbital (spatial variation of polarization) type of components. Recent research of OAM communication in radio frequency challenges the well-known MIMO technique for multi-channel encoding at single frequency [3], [4]. This encourages the RF community to investigate on possibilities of development, commercialization, and installation of compact antennas with OAM vortex beams at microwave frequencies.

Most of the research on the generation of LG-OAM beams were carried out at optical regime using Spiral Phase Plates [5], holograms [6], etc. However, due to the larger wavelength in microwave regime, designing efficient low profile antennas to generate OAM beams is a crucial issue. The concept of photon OAM was first developed by Thide et al. in the radio domain using a suitable arrangement of antennas [7]. Tamburini et al. used stepped spiral placement of Styrofoam covered with metallic foil at eight different steps to realize $2 \pi$ variation in phase [8] and vortex reflection. The same group came up with experimental demonstration of data transmission using sliced parabolic reflector [9]. Flat substrates with suitably drilled holes to transmit OAM vortex beam was successfully tested in millimeter [10] and terahertz [5] regime. Time switched antenna array [11], a circular arrangement of Vivaldi/patch antennas [12-14] with external phase shifting network was successfully tested for radio LG-OAM vortex beam generation. Recently, production of multimode vortex beams was tested using horn-fed planar reflectarray surface [15] and active element based phased surface [16]. Pancharatnam-Berry type principle to achieve phase variation through element rotation and generation of OAM beam by a spiral arrangement of the phased element was verified experimentally [17]. However, all the above works suffer from substantial structural dimension, complex feeding network (horn/phased array circuit) and complicated element distribution scheme. A unique concept of generating OAM vortex beam using a single circularly polarized MSA was reported in [18]. Also, recent work presented in [19] demonstrates the comprehensive radiation pattern synthesis of reflective phased surfaces. 
Metasurfaces, which are 2D counterparts of so-called metamaterials have a strong ability to enhance conventional volumetric wave-material interaction topologies [20]. Also, placement of surface elements for metasurface based applications became much more straightforward with the introduction of digital/coded distribution scheme [21]. Following which, a modified Jerusalem cross is studied and obtained phase is quantized to a 3-bit coding sequence in this paper. Based on that, two models of Phase Quantized Reflector Disks are proposed and realized. Simulated and experimental results at $\mathrm{x}$-band agree with the generation of OAM vortex mode.

\section{Meta-Element Synthesis and Phase Quantization}

As studied by Huygens, controlling the tangential components of field vectors one can fix the surface current which gives freedom to tailor the field outside the confined surface. Best practice to control that tangential component is to use suitable subwavelength secondary sources. Passive elements like stubs, patches, and metallic inclusions can introduce additional current density over the surface and dipole moments which leads to nonconventional field behavior.

To realize the meta-reflectors mentioned above, we need proper passive phase shifters with suitable phase swing and reflection efficiency. We studied most of the widely used reflectarray elements like square patch, slots, complementary structures, Jerusalem cross, etc. We noticed less than $320^{\circ}$ phase variation in all cases with unusual surface current at element edge which may cause a loss due to mutual coupling. Also, the phase variation curve was a steep response in many cases which may lead to root finding errors. Taking advantages from Jerusalem cross (appx $340^{\circ}$ phase tuning) and square ring (smooth response of phase with ring length) type elements we designed a modified element shown in Fig. 1 (a) in which a square ring is printed at the center of the Jerusalem cross to increase the current path and reduce overall element periodicity. The meta-element is made by thin copper printed over a grounded FR4 $(\varepsilon=4.3$ and thickness of $1.6 \mathrm{~mm})$. Here the width $w=3 \mathrm{~mm}$, the thickness of the copper film is $t=0.02 \mathrm{~mm}$, and the cell periodicity is $p=7 \mathrm{~mm}$. To realize the phase swing the length $l$ shown in Fig. 1(a) is varied from $1 \mathrm{~mm}$ to $3 \mathrm{~mm}$.

Finite element based solver of CST Studio ${ }^{\mathrm{TM}}$ is used to simulate and perform the reflection analysis of above meta-element. Periodic boundary conditions and tetrahedral meshing (mesh size of $0.02 \mathrm{~mm}$ min and $3.1 \mathrm{~mm}$ max) are adopted as pre-simulation settings for accurate results. Figure 1(b) depicts the reflection phase and magnitude for a linear variation of the parameter $l$ from $1 \mathrm{~mm}$ to $3 \mathrm{~mm}$. We can observe approximately $360^{\circ}$ variation in phase for all frequency samples considered above. Also, the phase response curve is of quasi-linear nature which reduces the
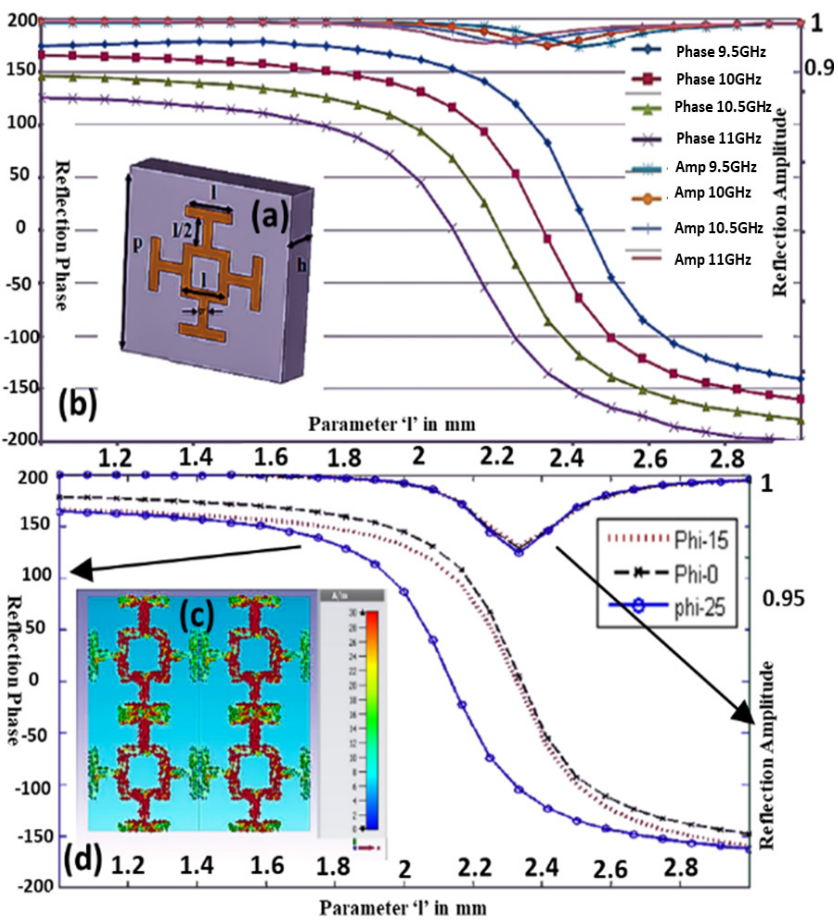

Fig. 1. (a) Geometry of the meta-element. (b) Phase and amplitude of reflection with variation of ' 1 ' for four frequency samples $(9.5 \mathrm{GHz}, 10 \mathrm{GHz}, 10.5 \mathrm{GHz}$ and $11 \mathrm{GHz}$ ). (c) The surface current distribution at $10 \mathrm{GHz}$. (d) Phase (deg) Y1-axis and amplitude Y2axis.

error of root finding in case of typical steep responses. The reflection magnitude is maximum (more than 0.9) for all simulations which promises a lossless reflector for almost $1.5 \mathrm{GHz}$ of bandwidth.

For further verification of the meta-atom, a parametric analysis for several incident angles is made at $10 \mathrm{GHz}$ using the same simulation settings. Figure 1(c) depicts the phase and amplitude curve for incident angle variation from $0^{\circ}$ to $25^{\circ}$ (three samples) and a negligible change in the nature of the responses is noticed in all cases. Displayed surface current (Fig. 1(d)) of the studied meta-element under periodic boundary confirms a stretch in the current path within the same unit cell dimension. Also at the edge of two consecutive elements, the current is negligible which avoids the errors due to mutual coupling. Due to smooth $369^{\circ}$ swing in phase, maximum reflection amplitude, huge incident angle tolerance and miniaturized element size, the studied meta-element can be implemented to design metasurfaces with adverse reflection functionalities in a broad frequency range of $9.5 \mathrm{GHz}-11 \mathrm{GHz}$.

Weather periodic or semi-periodic, the distribution of existing metamaterial or metasurface elements based on medium/surface transformation theory is always complicated due to complex parameter tensors. To simplify the placement of proper meta-elements in inhomogeneous engineered surfaces and mediums, several techniques are being proposed by investigators in the last decade. Digital or coding meta-elements or quantization of reflection/transmission parameters instead of complex medium properties 

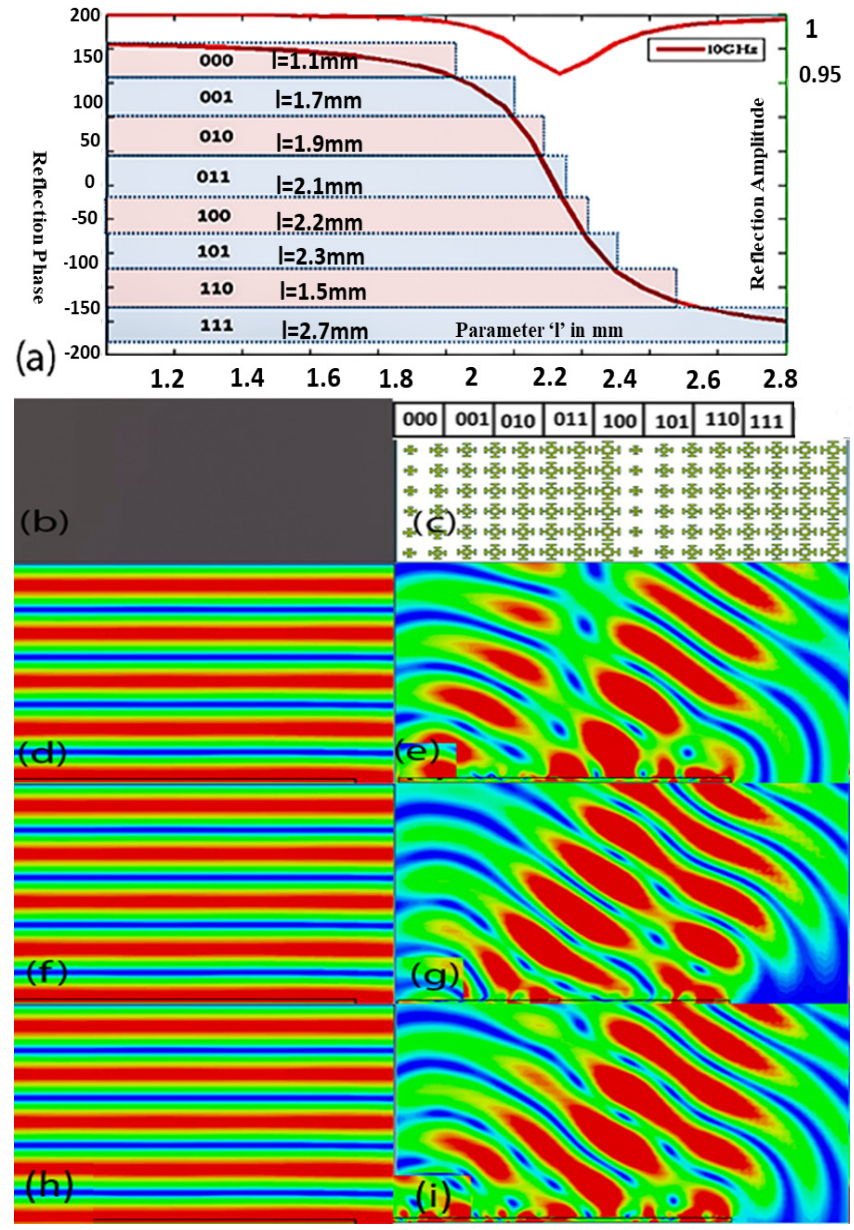

Fig. 2. Quantization of Meta-Atom Phase, (b) PEC surface, (c) coded surface, reflected E-field from (d, f and h) PEC surface and (e, g and i) from the coded surface at $9.5 \mathrm{GHz}, 10 \mathrm{GHz}$, and $10.5 \mathrm{GHz}$.

attracted the attention of the community from microwave to optical. Using coding metamaterial schemes proposed in optical regime [19] one can also investigate diversified applications of medium/surface engineering at radio regime.

In our work, the obtained meta-atom reflection phase from previous simulation result is discretized into 3-bit coding sequence. Eight quantized levels are chosen to represent the element parameter variation as digital bits. As shown in Fig. 2(a), each bit represents a link between reflection phase and the length $l$ of the meta-element at $10 \mathrm{GHz}$. From generalized laws of reflection [18], we can relate the angle of reflection and variation of surface phase as

$$
\begin{aligned}
& n \sin \theta_{\mathrm{r}}-n \sin \theta_{\mathrm{i}}=\frac{1}{k_{0}} \frac{\mathrm{d} \varphi}{\mathrm{d} x}, \\
& n \sin \theta_{\mathrm{r}}-n \sin \theta_{\mathrm{i}}=\frac{\lambda}{2 \pi} \frac{\mathrm{d} \varphi}{\mathrm{d} x} .
\end{aligned}
$$

Here $\theta$ is the angle of reflection/incident and $\lambda$ is the wavelength of the concerned medium. Without any phase change at the interface, the RHS of the above equation vanishes and conventional Snell's law will result. Now if we will take the medium like air and the infinitesimally small distance as $\mathrm{d} x=\lambda / 6$ and the phase change $\mathrm{d} \varphi=\pi / 4$ then equation (2) will give an angle of reflection of approximately $48^{\circ}$ for a normally incident wave. To realize this theory in practice, a surface consisting of $8 \times 16$ modified Jerusalem meta-atoms is designed whose surface phase varies from $+\pi \rightarrow-\pi$ two times (000 to 111 as phase quantization) along the $\mathrm{x}$-axis and remains symmetric along the y-axis. Simulations at several frequency samples are performed using finite integration solver of CST MWS and obtained scattered E-fields are compared with reflections from conducting plates. Figure $2(\mathrm{e}, \mathrm{g}, \mathrm{i})$ depicts the reflected field from linearly coded surface at $9.5 \mathrm{GHz}$, $10 \mathrm{GHz}$, and $10.5 \mathrm{GHz}$, respectively for a normal incident of plane wave which agrees with the theoretical study made above.

\section{Numerical and Experimental Verification of PQRD Models}

For OAM beam, components of Pointing vector travel helically around the direction of propagation. A blank core is created at the center due to the singularity in the propagation phase which leads to a conical beam shape. To realize such OAM beams in practice, we choose the element reflection method discussed above in which metasurface elements play the role of the subwavelength source to provide the desired phase shift. One can obtain the phase profile with these surface elements to produce desired LGOAM modes using the relation [15]

$$
\varphi_{i j}=-\frac{2 \pi}{\lambda}\left(r_{i j}-f_{i j}\right)+\arg \left\{\mathrm{e}^{\mathrm{j}\left(\frac{2 \pi}{\lambda} r_{i j} \pm l \alpha\right)}\right\} .
$$

Here $r_{i j}$ denotes the position vector of $i j$ th element of the $2 \mathrm{D}$ array, $l$ is the mode index of reflected vortex beam, $f_{i j}$ is the focal length between the source antenna and the element and $\alpha$ is the azimuth angle normal to the incident plane. To simplify the distribution reported in the abovecited article, we proposed two distinct coding sequences of meta-elements as shown in Fig. 3(a) and (b). These models represent sectorized and track-wise coding distributions, which generate mode of single arm vortex beam. Figures 3(c) and (d) display the simulated models of Phase Quantized Reflector Disks of $60 \mathrm{~mm}$ radius containing eight sectors and eight tracks, respectively. In Model-1 (sector based coding) each sector is filled with 30 metaatoms approximately whose values are selected from the phase quantization displayed in Fig. 2(a). The track-based coding (Model-2) has eight tracks (each track starts with bit ' 000 ' and ends with ' 111 ') and the number of elements in each track is $n=2 \pi r / p$ where $r$ represents the mean radius of each track and $p$ is the periodicity of the metaelement.

To reduce feed complexity and overall reflector di- 
mension, a rectangular microstrip patch antenna is considered as a source to incident quasi-spherical plane wavefront towards the PQRD. Adopting basic patch antenna dimensions, we used NELTEK substrate with thickness $0.75 \mathrm{~mm}$, dielectric constant of 3.2 and a patch dimension of $7.5 \mathrm{~mm} \times 10.2 \mathrm{~mm}$ for $10 \mathrm{GHz}$ (similar resonant frequency based dimension calculation scheme used for other frequencies).

In all frequency samples, the MSA is placed precisely $1 \lambda$ above the PQRD models. The educational license of CST-MW Studio ${ }^{\circledR}$ (Finite integration based solver) is used to simulate both the models using Perfect Boundary approximation (12 hexahedral mesh per wavelength).

Reflected E-field distributions depicted (an azimuthal frame of size $130 \mathrm{~mm} \times 130 \mathrm{~mm}$ ) in Fig. 4(a-c) are recorded
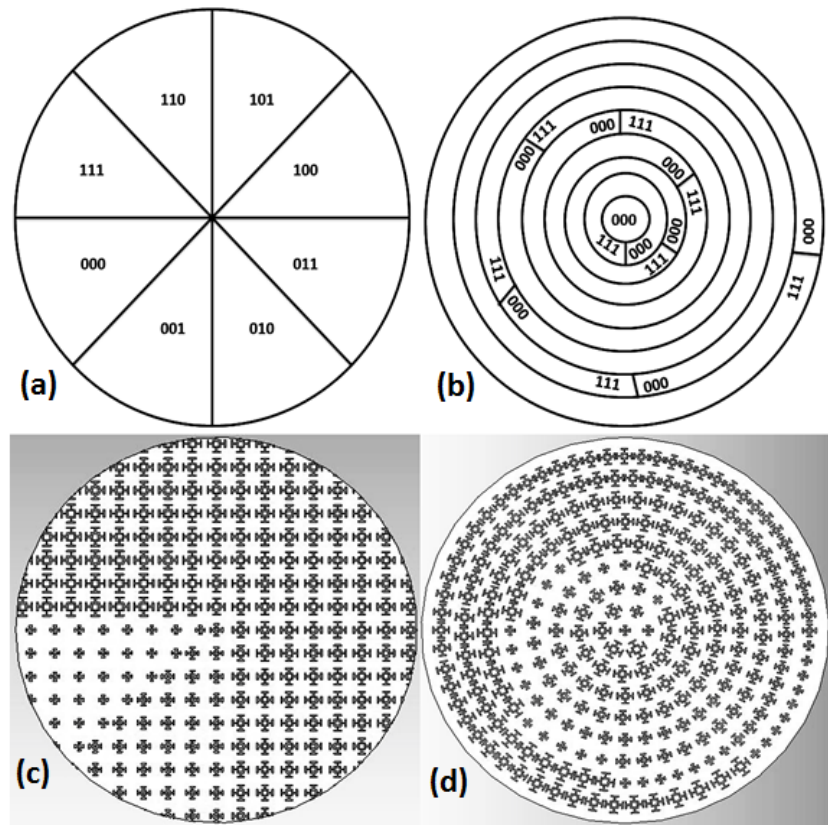

Fig. 3. Coded phase mask, simulation prototype: $(a, c)$. Model-1 and (b, d) Model-2.

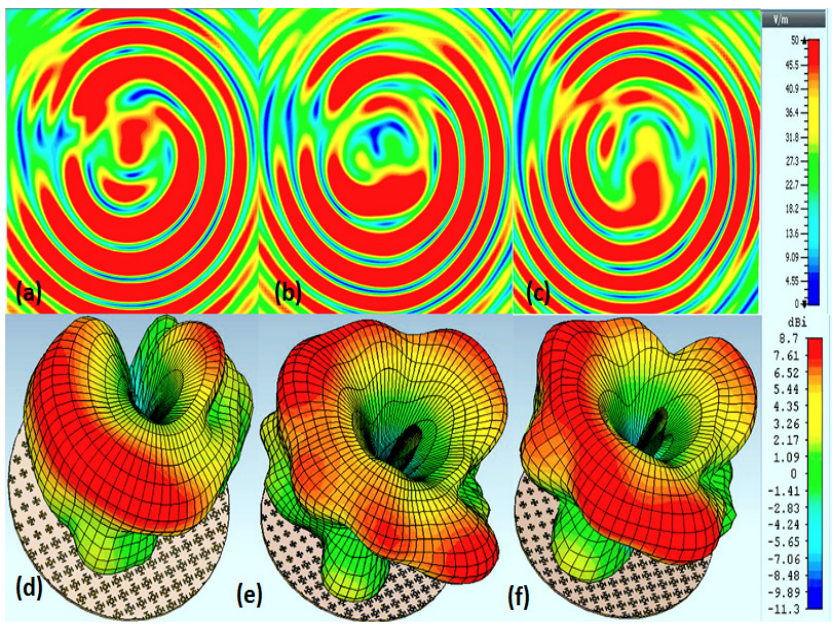

Fig. 4. (a-c) The E-field distribution at the azimuthal plane (d-f) The 3D far-field patterns at $(9.5 \mathrm{GHz}, 10 \mathrm{GHz}$ and $10.5 \mathrm{GHz}$ ) for Model-1 of PQRD.

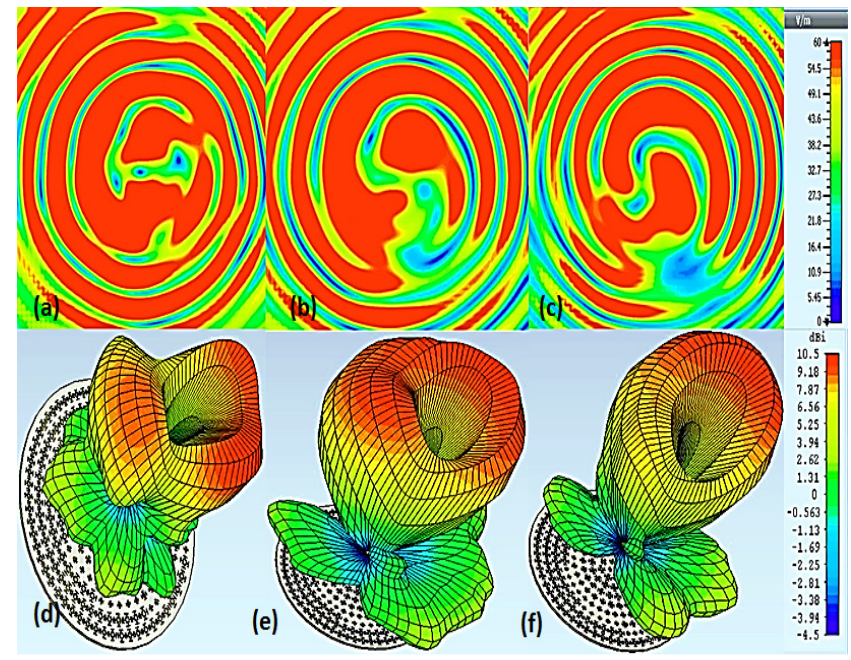

Fig. 5. (a-c) The E-field distribution at the azimuthal plane. (d-f) The 3D far-field patterns at $(9.5 \mathrm{GHz}, 10 \mathrm{GHz}$ and $10.5 \mathrm{GHz}$ ) for Model-2 of PQRD.

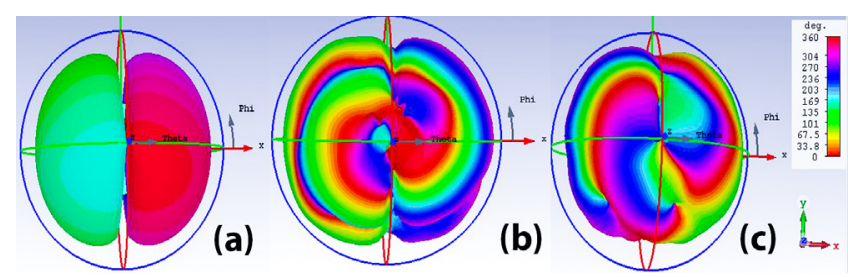

Fig. 6. Simulated radiated phase patterns of (a) rectangular microstrip patch antenna, (b) Model-1 and (c) Model-2 at $10.5 \mathrm{GHz}$

approximately $2 \lambda$ above the PQRD. The near-field results at three discrete frequencies are consistent with the above predicted primary mode of LG-OAM beam ensuring a broadband functionality of the reflector. From the farfield results displayed in Fig. 4(d-f), we can observe a dark core along propagation axis and an average maximum gain of $8.7 \mathrm{dBi}$ for all three cases.

Similar response of E-field for Model-2 of PQRD can be observed from Fig. 5(a-c) generating vortex mode at the azimuthal plane at a broad range of X-band. From the meta-element distribution of Model-2, we can notice the starting point of each track is random, which makes the phase distribution nonuniform in progressive radial direction. This improves the gain of far-field $2 \mathrm{dBi}$ more (Fig. 5(d-f)) than the previous model with zero radiation energy along the propagation axis. However, efficient nearfield and radiation behavior is noticed due to the linear variation of phase in each track of Model-2. Also from all simulated results, we saw a very minimum surface wave indicating low radiation loss. To strengthen the argument of generating Orbital Angular Momentum using proposed PQRD models, Figure 6 compares the radiated phase patterns of a bare MSA with the proposed models of PQRD. One can observe the helical phase profile which carries the OAM vortex beams. However, distortions are found due to discontinuity in phased element distribution.

Mits Autolab PCB prototyping machine is used to fabricate the proposed PQRD models and the source micro- 


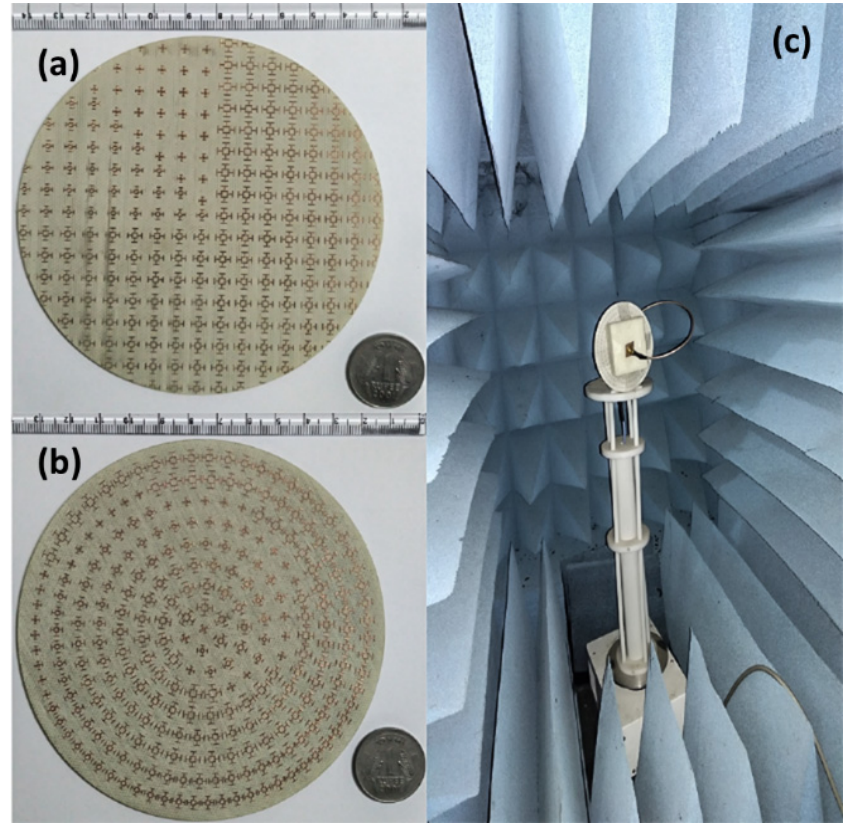

Fig. 7. (a, b) Fabricated prototypes of the PQRD models and (c) the experimental setup.

strip antennas. For each frequency sample, the MSAs are mounted precisely $1 \lambda$ above the PQRD models using ECOSTOCK PP foam with dielectric constant of 1.03 and permeability 0.99 . Figure $7(a, b)$ shows the prototypes of model-1 and model-2 of PQRD used for radiation performance analysis as a vortex generator. The measurement setup displayed in Fig. 7(c) is a $3 \mathrm{GHz}-12 \mathrm{GHz}$ anechoic chamber in which the metasurface reflector is mounted on a motor mast unit. Agilent PNA-L N5230A $(300 \mathrm{kHz}$ to $13.5 \mathrm{GHz}$ ) two-port network analyzer is used as a power supply and monitor unit. A standard x-band pyramidal horn is used as a reference antenna and placed approximately $3 \mathrm{~m}$ away from the antenna under test. For both the models of PQRD, the radiation properties are recorded at three frequency sample $(9.5 \mathrm{GHz}, 10 \mathrm{GHz}$, and $10.5 \mathrm{GHz})$ with a motor interval of $5^{\circ}$.

Comparison of measured and simulated normalized E-patterns of both PQRD models are displayed in Fig. 8 and Fig. 9. Measured results are recorded at two planes ( $\varphi=0^{\circ}$ and $\varphi=90^{\circ}$ ) to strengthen the prediction of the doughnut-shaped beam with dark core at the center. Approximate agreement is observed with simulated results in all comparison cases. As estimated, for both the models we are getting almost $-10 \mathrm{~dB}$ lower value of radiation at the center than those of the main beams which promises an efficient vortex beam generation. Also, the sidelobe levels are less than $-20 \mathrm{~dB}$ on average for all frequency samples. Moreover, discrepancies in radiation patterns are due to discontinuity of phase quantized sectors of the proposed models.

Based on a far-field experiment sample at $10 \mathrm{GHz}$, the working efficiency is estimated to strengthen the proposal of efficient vortex beam generation. Integrating the total scattering with and without PQRD, one can find the absorption energy $A$ which is $3.5 \%$ for Model-1 and 3.1\%
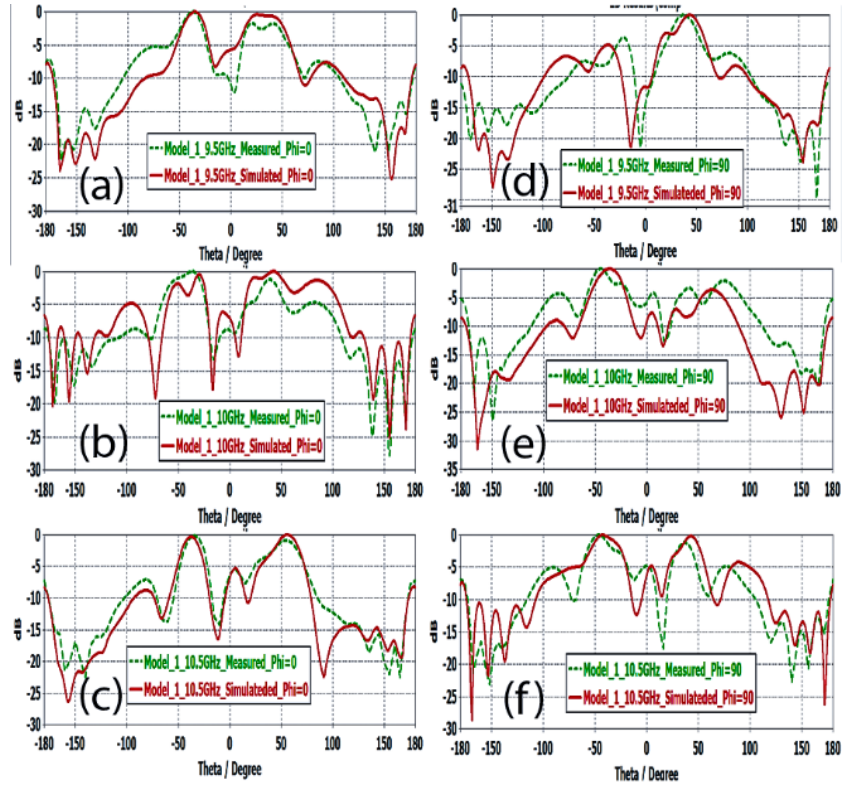

Fig. 8. Comparisons of simulated and measured normalized E-patterns for PQRD Model-1 (a-c) at $\varphi=0^{\circ}$ and (d-f) at $\varphi=90^{\circ}$ for $9.5 \mathrm{GHz}, 10 \mathrm{GHz}$, and $10.5 \mathrm{GHz}$.
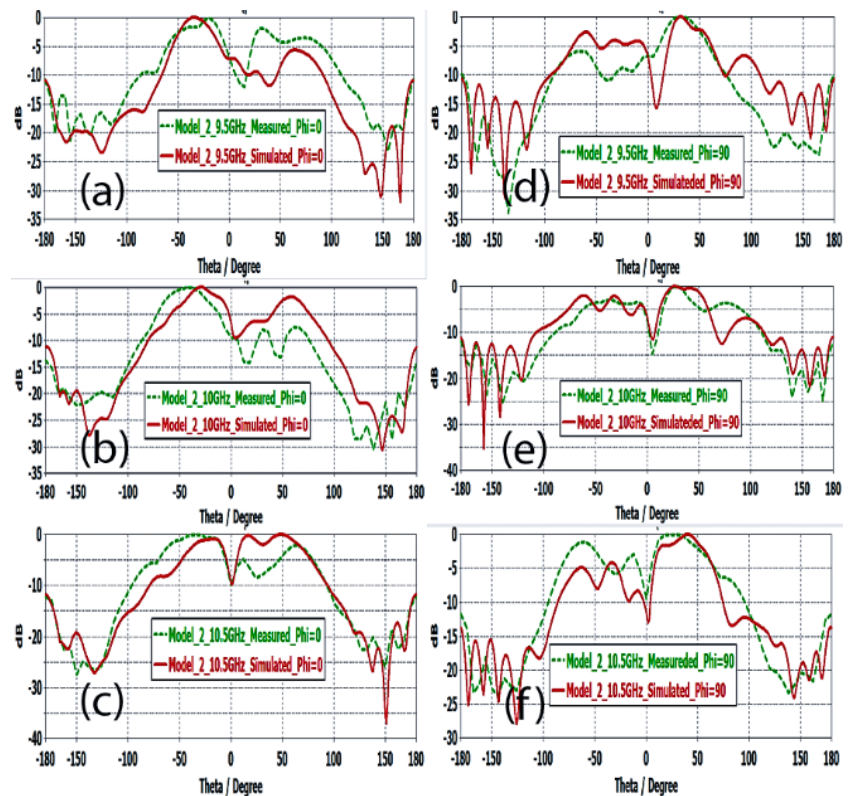

Fig. 9. Comparison of simulated and measured normalized E-patterns of PQRD Model-2 (a-c) at $\varphi=0^{\circ}$ and (d-f) at $\varphi=90^{\circ}$ for $9.5 \mathrm{GHz}, 10 \mathrm{GHz}$, and $10.5 \mathrm{GHz}$.

\begin{tabular}{|c|c|c|}
\hline Implementation method & Feeding technique & $\begin{array}{c}\text { Operating } \\
\text { frequency (GHz) }\end{array}$ \\
\hline Antenna array [12] & External network & 6 \\
\hline Spiral parabola [9] & Horn & 2.4 \\
\hline Flat surface with holes [10] & Horn & 30 \\
\hline $\begin{array}{c}\text { Thick metasurface (gap } \\
\text { between ground and } \\
\text { substrate) [15] }\end{array}$ & Horn & 5.8 \\
\hline Active metasurface [16] & Horn & 3.5 \\
\hline CP-MSA [18] & Coaxial connector & 2.4 \\
\hline $\begin{array}{c}\text { Phase coded surface } \\
\text { [This article] }\end{array}$ & MSA & $9.5-11$ \\
\hline
\end{tabular}

Tab. 1. List of contributions to generate LG vortex beams at radio frequencies. 
for Model-2 at $10 \mathrm{GHz}$. Similarly, we can estimate the transmission energy $T$ at $10 \mathrm{GHz}$ is $10.7 \%$ for Model- 1 and $11.2 \%$ for Model-2. From these data, we can calculate the working efficiency $(\% \sigma=100-\% T-\% A)$ for Model-1 and Model-2 of PQRD as $85.8 \%$ and $85.7 \%$ respectively at $10 \mathrm{GHz}$. Use of MSA as feed element reduces the overall size of the reflector disk whose diameter and thickness is around $11 \mathrm{~cm}$ and $3 \mathrm{~cm}$ respectively (much smaller than conventional horn-fed reflectarrays). List of contributions to generate LG vortex beams at radio frequencies is presented in Tab. 1 which indicates the evolution of proposed work using MSA as feed. This makes the proposed PQRD models suitable for movable platforms at X-band systems for realizing high channel capacity without increasing the bandwidth. In addition to that, information-reach radar, strategic devices, antijamming communication front-ends are few promises of the proposed PQRD models.

\section{Conclusion}

In this article, a modified Jerusalem type meta-atom is proposed to achieve approximately $360^{\circ}$ reflection phase swing. The phase is quantized into a 3-bit coding sequence based on which two models of metasurface reflector disks are proposed to generate OAM vortex beam at x-band. Rectangular MSA is used as a source for the PQRD models which reduced the feed complexity. Experimental results have good agreement with that of the numerical simulations. Approximately $85 \%$ radiation efficiency was recorded for both models. Simplified distribution scheme, miniaturized size, and reduction of feed complexity enables the use of the proposed antenna in radio OAM communication.

\section{Acknowledgments}

The authors are thankful to the Dept. of Science and Tech. Govt. of India for providing financial support to $\mathrm{Mr}$. R. Swain as INSPIRE Fellowship to peruse his doctoral program.

\section{References}

[1] ALLEN, L., BEIJERSBERGEN, M. W., SPREEUW, R. J. C., et al. Orbital angular momentum of light and the transformation of Laguerre-Gaussian laser modes. Physical Review A, 1992, vol. 45, no. 11 , p. 8185-8190. DOI: 10.1103/PhysRevA.45.8185

[2] WANG, J., YANG, J.-Y., FAZAL, I. M., et al. Terabit free-space data transmission employing orbital angular momentum multiplexing. Nature Photonics, 2012, vol. 6, no. 7, p. 488-496. DOI: $10.1038 /$ nphoton.2012.138

[3] MOHAMMADI, S. M., DALDORFF, L. K., BERGMAN, J. E., et al. Orbital angular momentum in radio - a system study. IEEE Transactions on Antennas and Propagation, 2010, vol. 58, no. 2, p. 565-572. DOI: 10.1109/TAP.2009.2037701
[4] EDFORS, O., JOHANSSON, A. J. Is orbital angular momentum (OAM) based radio communication an unexploited area? IEEE Transactions on Antennas and Propagation, 2012, vol. 60, no. 2, p. 1126-1131. DOI: 10.1109/TAP.2011.2173142

[5] CHENG, L., HONG, W., HAO, Z.-C. Generation of electromagnetic waves with arbitrary orbital angular momentum modes. Scientific Reports, 2014, vol. 4, p. 1-5. DOI: $10.1038 /$ srep04814

[6] CHÁVEZ-CERDA, S., PADGETT, M. J., ALLISON, I., et al. Holographic generation and orbital angular momentum of highorder Mathieu beams. Journal of Optics B: Quantum and Semiclassical Optics, 2002, vol. 4, no. 2, p. S52-S57. DOI: $10.1088 / 1464-4266 / 4 / 2 / 368$

[7] THIDÉ, B., THEN, H., SJÖHOLM, J., et al. Utilization of photon orbital angular momentum in the low-frequency radio domain Physical Review Letters, 2007, vol. 99, no. 8, p. 087701-1 to 087701-4. DOI: 10.1103/PhysRevLett.99.087701

[8] TAMBURINI, F., MARI, E., THIDÉ, B., et al. Experimental verification of photon angular momentum and vorticity with radio techniques. Applied Physics Letters, 2011, vol. 99, no. 20, p. 204102-1 to 204102-3. DOI: $10.1063 / 1.3659466$

[9] TAMBURINI, F., MARI, E., SPONSELli, A., et al. Encoding many channels on the same frequency through radio vorticity: first experimental test. New Journal of Physics, 2012, vol. 14, no. 3, p. 1-17. DOI: $10.1088 / 1367-2630 / 14 / 3 / 033001$

[10] BENISS, A., NIEMIEC, R., BROUSSEAU, C., et al. Flat plate for OAM generation in the millimeter band. In EuCAP2013- $7^{\text {th }}$ European Conference on Antennas \& Propagation. Gothenburg (Sweden), 2013, p. 3203-3207.

[11] TENNANT, A., ALLEN, B. Generation of OAM radio waves using circular time-switched array antenna. Electronics Letters, 2012, vol. 48, no. 21, p. 1365-1366. DOI: 10.1049/el.2012.2664

[12] DENG, C., CHEN, W., ZHANG, Z., et al. Generation of OAM radio waves using circular Vivaldi antenna array. International Journal of Antennas and Propagation, 2013, p. 1-7. DOI: $10.1155 / 2013 / 847859$

[13] WEI, W., MAHDJOUBI, K., BROUSSEAU, C., et al. Generation of OAM waves with circular phase shifter and array of patch antennas. Electronics Letters, 2015, vol. 51, no. 6, p. 442-443. DOI: $10.1049 / \mathrm{el} .2014 .4425$

[14] BAI, Q., TENNANT, A., ALLEN, B. Experimental circular phased array for generating OAM radio beams. Electronics Letters, 2014, vol. 50, no. 20, p. 1414-1415. DOI: $10.1049 / \mathrm{el} .2014 .2860$

[15] YU, S., LI, L., SHI, G., et al. Generating multiple orbital angular momentum vortex beams using a metasurface in radio frequency domain. Applied Physics Letters, 2016, vol. 108, no. 24, p. 241901-1-241901-5. DOI: 10.1063/1.4953786

[16] LI, C.-C., WU, L.-S., YIN, W.-Y. A dual-polarized and reconfigurable reflectarray for generation of vortex radio waves. AIP Advances, 2018, vol. 8, no. 5, p. 055331-1-055331-9. DOI: $10.1063 / 1.5023282$

[17] RAN, Y., LIANG, J., CAI, T., LI, H. High-performance broadband vortex beam generator using reflective Pancharatnam-Berry metasurface. Optics Communication, 2018, vol. 427, p. 101-106. DOI: 10.1016/j.optcom.2018.06.041

[18] BARBUTO, M., TROTTA, F., BILOTTI, F., et al. Circular polarized patch antenna generating orbital angular momentum. Progress In Electromagnetics Research. 2014, vol. 148, p. 23-30. DOI: 10.2528/PIER14050204

[19] ZHANG, D., CAO, X., YANG, H., et al. Radiation performance synthesis for OAM vortex wave generated by reflective metasurface. IEEE Access, 2018, vol. 6, p. 28691-28701. DOI: 10.1109/ACCESS.2018.2839099 
[20] YU, N., GENEVET, P., KATS, M. A., et al. Light propagation with phase discontinuities: generalized laws of reflection and refraction. Science, 2011, vol. 333, no. 6054, p. 333-337. DOI: $10.1126 /$ science. 1210713

[21] CUI, T. J., QI, M. Q., WAN, X., et al. Coding metamaterials, digital metamaterials and programmable metamaterials. Light: Science \& Applications, 2014, vol. 3, no. 10, p. 1-9. DOI: 10.1038/1sa.2014.99

\section{About the Authors ...}

Rajanikanta SWAIN (corresponding author) was born in India in the year 1990. He received his M.Sc. from Berhampur University India in 2012. He is perusing his doctoral program at Berhampur University, India under INSPIRE scheme of Govt. of India. His research interests include metamaterial, metasurface and reflectarray anten- nas. He is the recipient of IEEE MV Chauhan all India student paper contest for the year 2017.

Rabindra Kishore MISHRA was born in India in the year 1963. He is a professor in the Electronic Science Department of the Berhampur University. He has researched extensively in the areas of planar antennas and applications of soft computing techniques to analysis and design of planar antennas. He had visited the University of Birmingham as a British Commonwealth Fellow during 19992000. He has supervised 10 doctoral theses. He has published 2 monographs and over 150 learned articles in journals of repute and proceedings of conferences, seminars etc. These publications have earned the IETE Sir J. C. Bose best application paper award (1999) and Shri Hari Ohm Ashram Prerit Hariballabha Das Chunilal Research Endowment Award (2000), Samanta Chandra Sekhar Award in Engineering \& Technology (2008), which is the highest award by the Govt. of Orissa. 